\title{
Construction and evaluation of a whole genome microarray of Chlamydomonas reinhardtii
}

Jörg Toepel', Stefan P Albaum², Samuel Arvidsson ${ }^{3}$, Alexander Goesmann², Marco la Russa', Kristin Rogge ${ }^{1}$ and Olaf Kruse 1* $^{*}$

\begin{abstract}
Background: Chlamydomonas reinhardtii is widely accepted as a model organism regarding photosynthesis, circadian rhythm, cell mobility, phototaxis, and biotechnology. The complete annotation of the genome allows transcriptomic studies, however a new microarray platform was needed. Based on the completed annotation of Chlamydomonas reinhardtii a new microarray on an Agilent platform was designed using an extended JGI 3.1 genome data set which included 15000 transcript models.
\end{abstract}

Results: In total 44000 probes were determined (3 independent probes per transcript model) covering 93\% of the transcriptome. Alignment studies with the recently published AUGUSTUS 10.2 annotation confirmed 11000 transcript models resulting in a very good coverage of $70 \%$ of the transcriptome (17000). Following the estimation of 10000 predicted genes in Chlamydomonas reinhardtii our new microarray, nevertheless, covers the expected genome by $90-95 \%$.

Conclusions: To demonstrate the capabilities of the new microarray, we analyzed transcript levels for cultures grown under nitrogen as well as sulfate limitation, and compared the results with recently published microarray and RNA-seq data. We could thereby confirm previous results derived from data on nutrient-starvation induced gene expression of a group of genes related to protein transport and adaptation of the metabolism as well as genes related to efficient light harvesting, light energy distribution and photosynthetic electron transport.

\section{Background}

Chlamydomonas reinhardtii is widely accepted as a model organism regarding photosynthesis, circadian rhythm and biotechnology for several decades. With the first design of a C. reinhardtii microarray [1], transcriptomic analyses in this organism could be conducted. This first generation microarray contained 10000 transcript models with 8667 of them being associated with current transcript models covering about $87 \%$ of the predicted genome [2] with nearly 10000 genes. However, rapid progress in genome annotation [3] resulted in improved transcriptome data [4] which clearly demanded the design of a new microarray platform for advanced and general transcriptome analyses. Microarrays are relatively cheap and reliable systems to analyze transcript levels on a routine basis and they are perfectly complementary to

\footnotetext{
* Correspondence: olaf.kruse@uni-bielefeld.de

${ }^{1}$ Algae Biotechnology \& Bioenergy, Dept. of Biology, Center for

Biotechnology (CeBiTec), Bielefeld University, 33615 Bielefeld, Germany

Full list of author information is available at the end of the article
}

the recently established RNA-seq platforms [5]. The advantages of RNA-seq are manifold, e.g. the higher gene coverage and the increased sensitivity for differential gene expression [5-10]. The characterization of new gene models and splicing variations are easier to predict, as well as the detection and characterization of mutation sites [11]. However, the results of RNA-seq are still critical to examine and high reproducibility is often difficult to achieve. As a typical consequence, an overestimation of high abundant genes and length dependent amplification has been reported using RNA-seq [12-14]. These internal biases are still under discussion and data analysis and data normalization clearly need to be improved. Additional advantages of microarrays compared to RNAseq are still the significant lower costs (between 10-100 times) and the good coverage of exon based transcript levels, with around 90\% [13], where extremely deep sequencing would be necessary in order to achieve the same transcript coverage with RNA-seq. Furthermore, microarray experiments are less time consuming, allow
C Biomed Central 
the run of multiple replicates and established analysis platforms for routine transcript level analyses are available. However, the current microarray platform [1], with 10000 features, covers just $87 \%$ of the predicted genome and many newly annotated genes are missing [2]. Based on estimations using the Chlamydomonas genome, up to 17000 transcript models are expected to be present in this green algae $[4,15]$.

Generally, C. reinhardtii adaption to varying stress conditions can be best evaluated by using -omics approaches. Transcriptome studies were performed by different applications, e.g. microarray or RNA-seq, during nutrient starvation [16-18], anaerobiosis [19], hydrogen production [20], oxidative [21] or light stress [22]. The induction of genes responding to nutrient starvation, e.g. sulfur and nitrogen starvation is well documented and available data sets are used within this work to test the reliability of our newly designed microarray. A recent study investigating the effect of sulfur starvation [16] included a comparison of RNA-seq data with those acquired from microarray studies. The data comparison showed a good accordance between both methods. Adaptation to sulfur stress starts with an induction of genes responsible for nutrient transport accompanied by the repression of gene expression related to photosynthetic processes. In a later step, acclimatization/modulation processes include changes in the amino acid composition [23] of certain target proteins and the synthesis of starch [2]. Furthermore, lipid metabolism was shown to be affected under sulfur starvation caused by the physiological shift to anaerobic conditions $[23,24]$. In C. reinhardtii it is of particular interest that under sulfur depletion, plastidial hydrogenase activity and consequently hydrogen production is strongly induced $[25,26]$. Therefore, we also used existing transcriptome data sets of experiments to confirm gene expression pattern under sulfur starvation. So far, a number of studies employing high-throughput technologies including transcriptomics, proteomics and metabolomics have been carried out to describe the process of hydrogen production in C. reinhardtii $[20,24,27]$.

Another well documented stress condition is the growth of $C$. reinhardtii under nitrogen starvation [28-30]. Recent analyses of gene expression variation under nitrogen starvation by RNA-seq [17] precisely describe adaptation processes of photosynthesis as well as of anabolic metabolism mechanisms (lipid and amino acid production). The high sensitivity of RNA-seq was demonstrated by monitoring differences in expression rates of low abundant genes coding for transcripts involved in regulatory processes. In detail, it could be concluded that nitrogen starvation results in a decreased photosynthetic gene expression and activity, increased lipid accumulation and induction of gametogenesis.
In this present study, we performed microarray experiments with our newly developed $C$. reinhardtii full genome microarray to prove its suitability for differential transcript analyses and for comparing time-course global expression profiles of $C$. reinhardtii under starvation conditions. Additionally, we tested the sensitivity of the array for identifying knockout mutations.

\section{Results \\ Design}

Microarray design was based on data sets provided by the JGI 3.1 genome annotation of $C$. reinhardtii. This new Chlamydomonas reinhardtii microarray platform is now available under the Agilent ${ }^{\odot}$ access number 024664. The microarray design is, however, not fixed: a crucial advantage is that newly annotated genes can easily be added to this array. The adapted new transcriptome consists of 15000 annotated nucleus-encoded gene models. We designed 60 mer oligonucleotides using two software tools: ARRAY-EXPRESS ${ }^{\odot}$ and PROMIDE $^{\odot}$. The detailed origin for each probe and probe sequence is summarized in additional file 1, Table S1. Both software tools were used to design temperature and position optimized probes (5'-3' bias). As a result, we determined sequence optimized probes for 14557 gene models, which represent $93 \%$ coverage of the transcriptome based on the JGI3.1 genome annotation. We were unable to determine specific probes for the remaining transcript models. In Figure 1, the chemical properties of the designed probes are described. The G/ $C$ content of the probes varied between 40 and $60 \%$ (Figure 1A) and melting temperature distribution for all probes was between 80 and $90^{\circ} \mathrm{C}$, (Figure $1 \mathrm{~B}$ ).

Additionally, we used 8760 approved sequences from the first generation $C$. reinhardtii microarray, adapted to the Agilent ${ }^{\odot}$ platform [3] and added the probes as a third replicate to our microarray platform. However, according to our BLAST analysis (reference: AUGUSTUS 10.2) are just 7200 probes specific for one transcript model and we used just proved probes for our new microarray platform.

Finally, we compared the new determined probe sequences with the new published annotation (AUGUSTUS 10.2) and could confirm in silico by BLAST analysis http://blast.ncbi.nlm.nih.gov/ the hybridization specificity for $70 \%$ of the 14557 transcript specific sequences. The remaining probes showed potential cross hybridization properties (7\%, with more than 3 mismatches in the sequence) or could not be aligned to current transcripts (23\%). It should be noted that the genome annotation of the $C$. reinhardtii genome is not yet finished and the final number of transcript models is still under discussion. Detailed information about the specificity and potential 

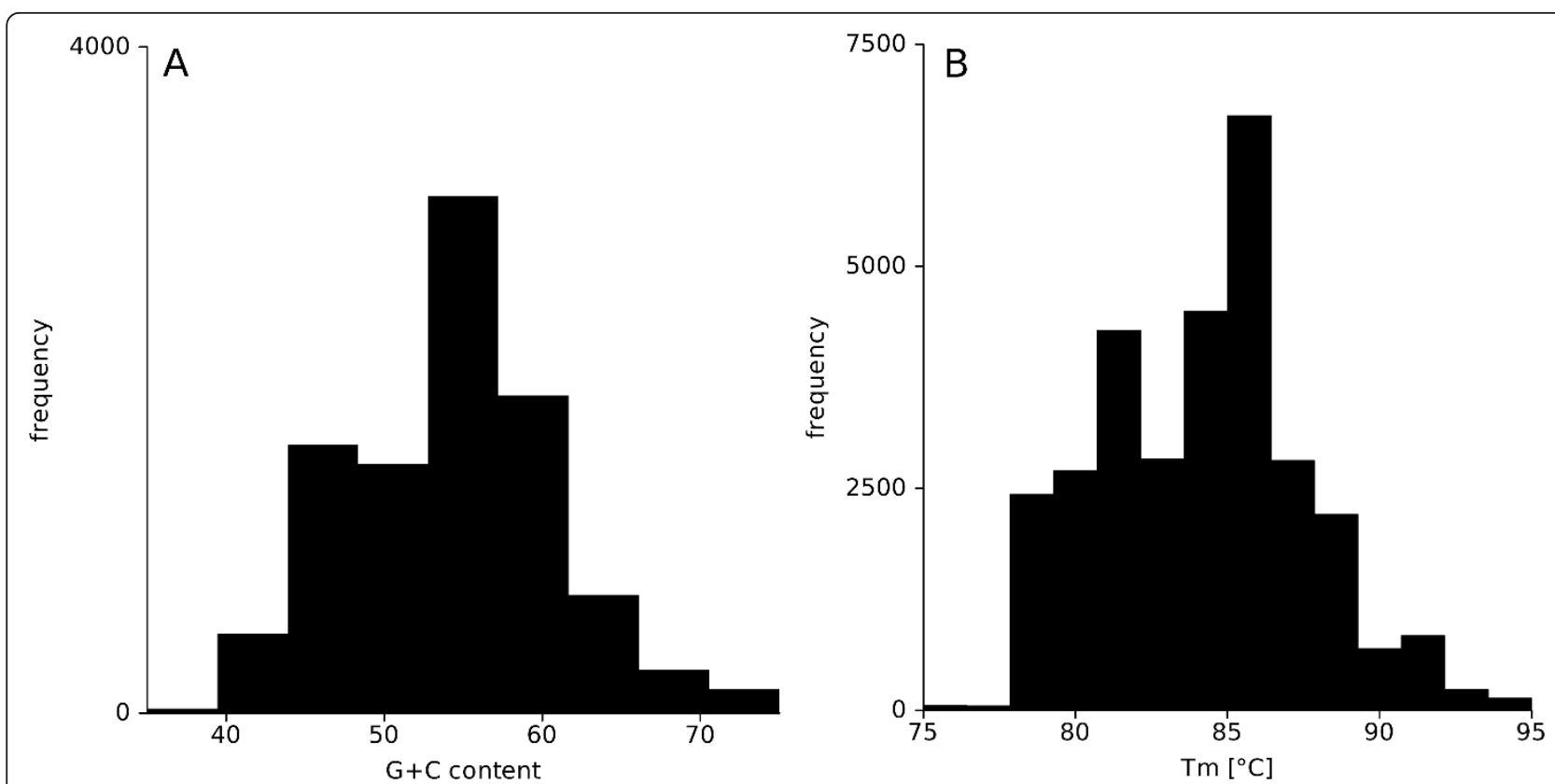

Figure 1 Chemical properties of the designed transcript probes for a Chlamydomonas reinhardtii $4 * 44 \mathrm{k}$ Agilent microarray: (A) Histogram of the G+C content and (B) Histogram of the melting temperature (Tm).

cross hybridization targets is provided in additional file 2, Table S2.

\section{Testing}

We used identical RNA samples from cells grown for 24 $\mathrm{h}$ under sulfur starvation to check $\mathrm{Cy}-3$ and $\mathrm{Cy}-5$ labeling. Successful pre-correction was achieved with the feature extraction software (10.7.3.1). As a result we could demonstrate that labeling is nearly identical with both dyes. The $\mathrm{Cy} 3 / \mathrm{Cy} 5 \log 2$ ratio showed a good distribution around 0 and therefore an additional dye-specific correction is not necessary (Figure 2). To ensure that any remaining differences between the two labeling dyes are equalized, all computed $\log 2$ ratios were normalized. Following the recommendations of [31], a normalization method based on robust local regression (lowess) was utilized for this purpose.

Normalization and analysis were carried out with the in-house developed EMMA2 software [32]. To test the reproducibility of the data, a 6 fold replication with labeled RNA samples taken from starvation experiments was performed. The high similarity of the data sets with a $\log 2$ ratio variation between 20 and 35\% for all differential expressed genes derived from three biological and three technical replicas demonstrated the robustness of the system (Figure 3). The internal C. reinhardtii specific control probes showed a variation smaller than $10 \%$ (data not shown) in all experiments and could be therefore considered as a reliable control parameter for further experiments.

\section{Starvation experiments}

We performed sulfur and nitrogen starvation experiments with $C$. reinhardtii WT cc3491 to analyze the expression of genes responding to nutrient starvation. As a result 25000 probes showed a significant fluorescence signal against the background, and were

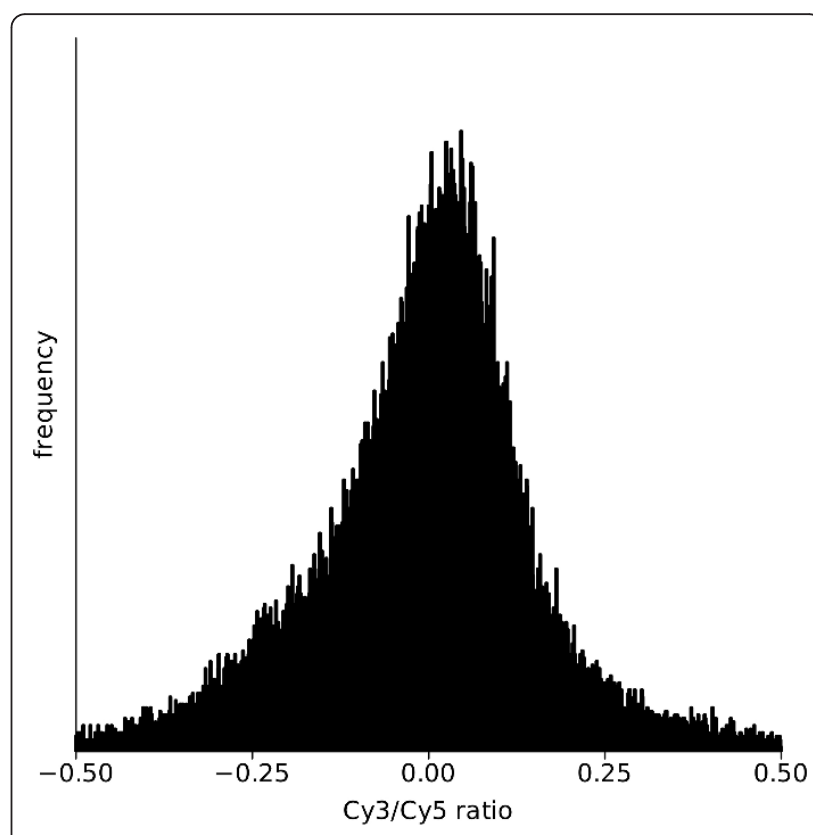

Figure 2 Testing of the $\mathrm{Cy}-3$ and $\mathrm{Cy}-5$ labeling pre-correction with the feature extraction software (10.7.3.1.), using identical RNA samples of Chlamydomonas reinhardtii. 


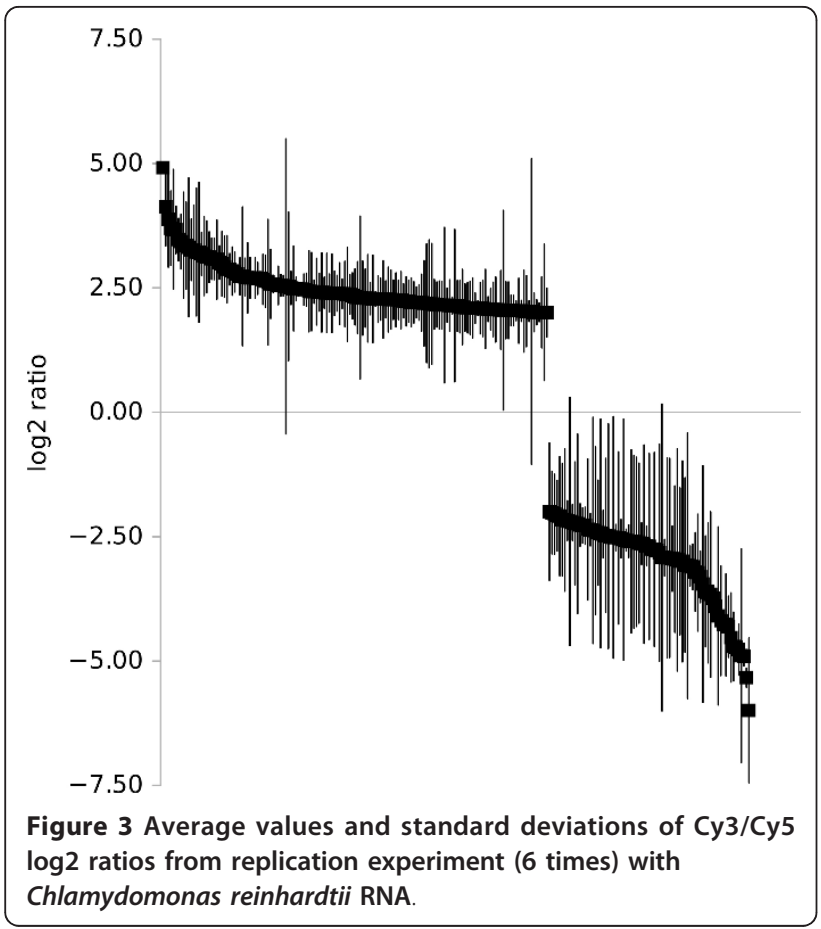

therefore used for data analysis. With three independent probes per transcript, around 8000 transcript models could be analyzed in the experiments. It should be noted that results derived from the newly designed probes showed a good concordance. However data derived from the probes of the first generation array showed a lower $\log 2$ ratio in differentially expressed genes and blast analysis showed a high cross hybridization potential for many probes. We therefore decided not to include these data sets into the analysis. The data analysis resulted in the identification of a relatively small number of genes responding to the respective nutrient limitation conditions. We performed a cluster analysis using the software GENESIS ${ }^{\odot}$ (hierarchical cluster analysis using Ward and Euclidean distances) for differentially regulated genes, to detect time-dependent gene expression in response to nutrient stress, and could conclude that the majority of the genes showed a fast response to the nutrient stress with constant expression level over the whole timecourse. The late-responding genes mainly belong to transcripts related to secondary effects like phosphorus stress or $\mathrm{CO}_{2}$ limitation.

\section{Nitrogen starvation}

The response of gene expression during nitrogen starvation was in general higher and faster than during sulfur starvation conditions. Around 200 genes showed twofold increased expression levels whereas approximately 300 genes were detected with a twofold reduced expression (additional file 3, Table S3). Comparison with RNA-seq transcriptome data derived from nitrogen starvation experiments [17] revealed a very similar picture with an identical differential gene expression pattern for $60 \%$ of the transcripts. In [17] transcripts were analyzed using Illumina Solexa ${ }^{\odot}$ combined with the $454^{\odot}$ ultra-fastsequencing which resulted in the identification of several more transcripts. Differences in the two datasets are most likely a result of differences in time length of starvation (72 $\mathrm{h}$ instead of $48 \mathrm{~h}$ ) and of differences in the intensity of illumination $\left(200 \mu \mathrm{mol} \mathrm{m} \mathrm{m}^{-2} \mathrm{~s}^{-1}\right.$ instead of 80 $\mu \mathrm{mol} \mathrm{m}{ }^{-2} \mathrm{~s}^{-1}$ ). Another reason could be the possible bias by overestimation of large transcripts and differences in the range of detection. Within our experiments we were able to confirm the up-regulation of components of the nitrogen transport systems, such as ammonia-, nitrateand nitrite-transporters (see Table 1). From 7 annotated ammonium transporters (AMT), only AMT4 and AMT1 were up-regulated. This result was achieved with all three probes per transcript with a minimal $\log 2$ ratio of 4 and shows high similarities to earlier data provided by [17], however here some of the AMT transcripts were most likely miss-annotated. The nitrate transporter with the highest expression level was encoded by the locus Cre09.g410850.t1.1 As expected, the major nitrate transporter did not response to nutrient stress, since ammonia was provided as the nitrogen source in the growth medium. Furthermore, the up-regulation of one of the four annotated glutamine synthetases (GLN3, Cre12. g530600.t1.1) was confirmed, no up-regulation of GLN1 (Cre02.g113200.t1.1); GLN2 (Cre12.g530650.t1.1) and GLN4 (Cre03.g207250.t1.1) was detectable, similar results were obtained from the RNA seq data. Additionally, increased gene expression for one nitrite reductase (Cre09.g410750.t1.1) and one nitrate reductase (Cre09. g410950.t1.1) could be confirmed within our experiments. Interestingly, although under these conditions the majority of the photosynthetic gene transcripts were down-regulated, several genes related to photosynthesis showed an up-regulation in transcription rates. In detail, some genes responsible for PS light harvesting and energy distribution like LHCA1, LHCSR3 and several photosystem II subunits like $P S B X$ and $P S B S 1$ were upregulated during nitrogen starvation. However, it is noteworthy and somewhat surprisingly that we detected at the same time down-regulation of LHCSR1 under nitrogen limitation, a result which is in good accordance to RNA-seq data. It has been reported that $L H S C R$ genes are up-regulated under stress conditions and responsible for de-excitation of chlorophyll molecules in PS II [33,34]. Furthermore in good agreement with [17], none of the ribosomal related genes were up-regulated. We could further confirm the NIT2 induction (transcription factor regulating nitrogen metabolism) and the 
Table 1 Comparison between RNA-seq [17] (48 h) and microarray data for Chlamydomonas reinhardtii cultures incubated under nitrogen starvation conditions For microarray data average values for important genes during $96 \mathrm{~h}$ starvation are displayed ( $24 \mathrm{~h}, 48 \mathrm{~h}, 72 \mathrm{~h}, 96 \mathrm{~h})$.

\begin{tabular}{|c|c|c|c|c|}
\hline $\begin{array}{l}\text { Reporter } \\
\text { Identifier }\end{array}$ & $\begin{array}{l}\text { Reporter } \\
\text { Name }\end{array}$ & Reporter Description & $\begin{array}{l}\text { Log2 ratio } \\
\text { microarray }\end{array}$ & $\begin{array}{l}\text { Log2 ratio RNA } \\
\text { seq }\end{array}$ \\
\hline & \multicolumn{4}{|c|}{ nitrogen related genes } \\
\hline 182971 & NSG13 & "nitrogen-starved gametogenesis $13^{\prime \prime . . . ~}$ & 5.0 & 3.6 \\
\hline 184661 & NIT1 & "nitrate reductase"... & 4.6 & 7.9 \\
\hline 192085 & NII1 & "Nitrite reductase"... & 4.4 & 7.7 \\
\hline 205647 & NIT2 & "transcription factor regulating nitrogen metabolism"... & 1.5 & 2.6 \\
\hline 188890 & & "NADH nitrate reductase"... & 3.0 & 5.2 \\
\hline 156131 & AMT4 & "Ammonium transporter"... & 5.1 & 7.2 \\
\hline 158745 & AMT1 & "Ammonium transporter"... & 3.1 & 5.5 \\
\hline 133971 & GLN1 & Glutamine synthetase & 0.9 & 1 \\
\hline 129468 & GLN2 & Glutamine synthetase & 0.1 & -0.3 \\
\hline 136895 & GLN3 & Glutamine synthetase & 6.5 & 6.6 \\
\hline \multirow[t]{2}{*}{147483} & GLN4 & Glutamine synthetase & 2.3 & 1 \\
\hline & \multicolumn{4}{|c|}{ photosynthetic related genes } \\
\hline 184724 & LHCSR1 & "stress-related chlorophyll a/b binding protein $1 " \ldots$ & -2.4 & -1.2 \\
\hline 184731 & LHCSR2 & "Stress-related chlorophyll a/b binding protein 2"... & 3.7 & 2.6 \\
\hline 184730 & LHCSR3 & "Stress-related chlorophyll a/b binding protein 3"... & 3.8 & 1.7 \\
\hline 196341 & PSBS1 & "chloroplast Photosystem II-associated 22 kDa protein"... & 2.0 & 6.9 \\
\hline 171516 & PSBS2 & "chloroplast Photosystem II-associated 22 kDa protein"... & -0.3 & \\
\hline 196341 & PSBX & "4.1 kDa photosystem II subunit"... & -2.1 & 6.9 \\
\hline 205940 & PSAL & "Photosystem I reaction center subunit XI"... & -2.4 & 0.0 \\
\hline 192478 & PSAK & "photosystem I reaction center subunit psaK"... & -2.3 & -3.4 \\
\hline 184810 & LHCB4 & "chlorophyll a-b binding protein of photosystem II"... & -2.4 & -2.3 \\
\hline 187025 & LHCA8 & "light-harvesting protein of photosystem I"... & -2.2 & -4.6 \\
\hline 192961 & LHCA7 & "light-harvesting protein of photosystem I"... & -2.5 & -5.0 \\
\hline 186299 & LHCA6 & "light-harvesting protein of photosystem I"... & -2.7 & -5.4 \\
\hline 153678 & LHCA4 & "light-harvesting protein of photosystem I"... & -2.6 & -4.5 \\
\hline 206001 & LHCA1 & "light-harvesting protein of photosystem I"... & -3.2 & \\
\hline 184397 & LHCBM3 & "Light-harvesting complex II chlorophyll a-b binding protein M3"... & -2.0 & -3.7 \\
\hline 184479 & LHCBM9 & "chlorophyll a-b binding protein of LHCII"... & -0.2 & -1.5 \\
\hline \multirow[t]{2}{*}{126810} & NAB1 & "nucleic acid binding protein"... & -1.6 & -4.1 \\
\hline & \multicolumn{4}{|c|}{ other genes } \\
\hline 185841 & RDP3 & "Rhodanese domain phosphatase"... & 2.4 & -3.4 \\
\hline 196438 & PTB5 & "sodium/phosphate symporter"... & 5.3 & 0.6 \\
\hline 196465 & PTB5 & "sodium/phosphate symporter"... & 4.3 & 1.7 \\
\hline 183357 & PTB3 & "sodium/phosphate symporter"... & 3.8 & -0.6 \\
\hline 96789 & GPD4 & $\begin{array}{l}\text { "Glycerol-3-Phosphate Dehydrogenase/Dihydroxyacetone-3-Phosphate } \\
\text { Reductase"... }\end{array}$ & 2.8 & 1.4 \\
\hline 146945 & GPD1 & $\begin{array}{l}\text { "Glycerol-3-Phosphate Dehydrogenase/Dihydroxyacetone-3-phosphate } \\
\text { Reductase"... }\end{array}$ & 3.6 & 4.1 \\
\hline 182461 & GLD2 & "glucose-6-phosphate dehydrogenase"... & 2.3 & 4.1 \\
\hline 191987 & ARG1 & "N-acetyl-gamma-glutamyl-phosphate reductase"... & 2.8 & 0.8 \\
\hline R:191668/1 & ICL1 & "isocitrate lyase" & 0.1 & -5.8 \\
\hline
\end{tabular}

repression of $N A B 1$, a nuclear encoded mRNA binding factor, which specifically binds and sequesters $L H C I I$ mRNA and prevents their translation [35-37]. Many carbonic anhydrases showed a down-regulation; however the mitochondrial carbonic anhydrase (Cre05.g248450. t1.1) was up-regulated. Regarding the TCA-cycle the transcript level of the citrate synthase (Cre12.g514750. t1.1) increased during nitrogen starvation, in contrast to the isocitrate lyase 1 (ICL1) which was not affected in our experiments. Additionally, our data indicated an upregulation of several genes induced by phosphate starvation, like PSR1 (phosphorus starvation response 1 
protein, transcriptional regulator) as earlier described [18]. PSR1 however, did not show an increased level of expression within the RNA-seq data. Again, the reason for the differences could be due to the longer starvation period leading to secondary effects.

\section{Sulfur stress}

Under sulfur starvation 813 genes showed a differential gene expression. 300 genes were down-regulated by at least 2-fold whereas around 100 genes were at least 2fold up-regulated. Comparison between first generation and new microarray data derived from sulfur starvation experiments showed high similarities for many genes regarding changes in their differential gene expression pattern (for details see additional file 4, Table S4). We confirmed the up-regulation for several sulfate transport systems and induction of several stress response systems (Table 2). As shown in Figure 4 we were able to confirm the increased gene expression of two major aryl sulfatases (ARS1 and ARS2). Probe specificity for the other ARS enzymes could be confirmed but no increased gene expression was detectable. It should be noted that for ARS6, no probes were designed, since this gene has not yet been annotated at the time of the experiment. The extracellular proteins ECP88, ECP76, SLP3 (Sulfate binding protein), SUA (Chloroplast sulfate transporter) STL1 (sodium/sulfate co-transporter) and SIR1 (ferredoxin sulfite reductase 1) also showed an up-regulation for all determined and tested probes. Similar to the experiments with nitrogen starvation, most photosynthetic related genes were down-regulated however for several proteins involved in light harvesting and light quenching (LHSBM9, LHCSR1, LHSCR3.1 and LHSCR3.2) we could confirm an increase in transcript levels during all 4 time points.

Overall, we found the majority of the genes $(60 \%)$ previously described to be induced or repressed by sulfate starvation with the same expression pattern [16]. Variations can be explained by the different time scale of sample harvesting and different growth conditions which could also lead to secondary effects like phosphate limitation. Differences in the $\log 2$ ratios between RNA-seq data and microarray data are most likely a result of the higher dynamic range of the RNA-seq data, since saturation effects, which can occur for highly expressed genes on microarrays, are not expected.

Combing the array data sets derived from the two starvation experiments we identified several genes, which showed an increased or reduced transcript level under both nutrient stress conditions (additional file 5, Table S5). Most of these genes are either of unknown function or are reported to be involved in transport or metabolism.

\section{LHCSR3 knock out detection}

To analyze the specificity of the array system we used the knock out mutant $n p q 4$ (kind gift of Prof. Krishna Niyogi, University of California), which has been previously shown to be deficient in the LHCSR3 gene transcripts [38], to check if we can detect the genotype on the RNA level. It is known that the LHSCR1, LHCSR3.1 and LHSCR3.2 gene expression is enhanced under sulfur starvation and/or during hydrogen production induced by sulfur deprivation [23]. Therefore, we analyzed the transcript level for $n p q 4$ and the parental strain $4 A+$ under sulfur starvation. The expression rates under sulfate starvation increased for all three transcripts in the wild type, but no gene expression was detectable in the npq4 mutant in the LHCSR3.1 and LHSCR3.2 isoforms, as expected (see Figure 5). For this reason, we can state a high specificity for our designed probes regarding the LHCSR transcripts and in combination with the analyzed nutrient starvation experiments a good usability of our microarrays. Comparison with WT cc3491 grown under sulfur deprivation, showed no significant changes regarding the normal response to sulfur starvation.

\section{Discussion}

In this work we successfully designed and tested a novel microarray platform for Chlamydomonas reinhardtii. We were able to determine unique sequences for most (93\%) of the transcript models obtained from the Joint Genome Institute data base version JGI 3.1. For a small number of transcript models (7\%) we could not identify a specific gene sequence and consequently these genes cannot be analyzed with the current microarray. We confirmed the specificity for 11000 probe sets with the current annotation (AUGUSTUS 10.2). We tested our microarray with RNA samples from cultures grown under different nutrient stress conditions and compared our data with recent publications. By doing this, we could confirm with our new system previously published changes in gene expression during nutrient starvation for many genes and hereby proved that this newly designed array is very useful for general transcription analysis. In addition, RNA amplification enabled us to detect several low abundant regulator genes expressed under nutrient starvation with the acquired data being in good accordance with previously published RNA-seq and microarray data [16,17]. Microarrays are cheap and reliable tools for monitoring transcript changes, and although RNA-seq methods may allow a more detailed view inside the transcriptome, the lower costs, the high reproducibility and the established analysis systems advantages microarrays for routine applications. Therefore, one can now choose the platform, which provides the best conditions for the individual experiment. 
Table 2 Comparison between RNA seq [16] (24 h) and microarray data for Chlamydomonas reinhardtii cultures incubated under sulfur starvation conditions For microarray data average values for the genes during $96 \mathrm{~h}$ starvation are displayed ( $24 \mathrm{~h}, \mathbf{4 8} \mathrm{h}, \mathbf{7 2} \mathrm{h}, \mathbf{9 6} \mathrm{h})$.

\begin{tabular}{|c|c|c|c|c|}
\hline Reporter Identifier & Reporter Name & Reporter Description & Log 2 ratio microarray & Log 2 ratio RNA seq \\
\hline & \multicolumn{4}{|c|}{ sulfur related genes } \\
\hline 59800 & SUOX1 & "sulfite oxidase"... & 3.5 & 2.0 \\
\hline 205505 & SLT3 & "sodium/sulfate co-transporter"... & -4.5 & -1.8 \\
\hline 205506 & SLT2 & "sodium/sulfate co-transporter"... & 2.1 & -1.7 \\
\hline 205507 & SLT1 & "sodium/sulfate co-transporter"... & 3.1 & 6.9 \\
\hline 206159 & $\mathrm{SIR} 1$ & "ferredoxin-sulfite reductase"... & 1.7 & 2.0 \\
\hline 55757 & ARS2 & "periplasmic arylsulfatase"... & 5.4 & 8.2 \\
\hline 205501 & ARS1 & "periplasmic arylsulfatase"... & 4.3 & 6.3 \\
\hline 133924 & ATS2 & "ATP-sulfurylase"... & 4.0 & 2.8 \\
\hline 175651 & \multicolumn{2}{|c|}{ OASTL1 changed to ASL1 } & -1.2 & -0.3 \\
\hline 137329 & ECP88 & "88 kDa extracellular polypeptide"... & 5.6 & 13.4 \\
\hline \multirow[t]{2}{*}{130684} & ECP76 & "76 kDa extracellular polypeptide"... & 5.5 & 10.7 \\
\hline & \multicolumn{4}{|c|}{ photosynthetic genes } \\
\hline 182015 & PSBX & "4.1 kDa photosystem II subunit"... & -3.1 & -0.9 \\
\hline 193847 & PSAO & "Photosystem I subunit $\mathrm{O}^{\prime . . .}$ & -3.5 & -1.4 \\
\hline 182959 & PSAH & "Subunit H of photosystem I"... & -3.1 & -1.4 \\
\hline 187195 & PRPL29 & “Putative chloroplast ribosomal protein L29 & -7.0 & -2.3 \\
\hline 184730 & LHCSR3 & "Stress-related chlorophyll a/b binding protein 3"... & 3.9 & 2.3 \\
\hline 184731 & LHCSR2 & "Stress-related chlorophyll a/b binding protein 2"... & 2.4 & 2.1 \\
\hline 184724 & LHCSR1 & "stress-related chlorophyll a/b binding protein 1"... & 5.5 & 1.8 \\
\hline 184479 & LHCBM9 & "chlorophyll a-b binding protein of LHCII"... & 5.8 & 10.0 \\
\hline 205757 & LHCBM8 & "chlorophyll a-b binding protein of LHCII"... & -2.8 & -0.5 \\
\hline 184071 & LHCBM7 & "chlorophyll a-b binding protein of LHCII"... & -2.6 & -0.9 \\
\hline 191690 & LHCBM4 & "chloropyll a-b binding protein of LHCII"... & -2.9 & -0.3 \\
\hline 184397 & LHCB5 & "minor chlorophyll a-b binding protein of photosystem ॥" & -3.2 & -2.0 \\
\hline 187025 & LHCA8 & "light-harvesting protein of photosystem I"... & -2.9 & -1.3 \\
\hline 183363 & LHCA7 & "light-harvesting protein of photosystem I"... & -3.5 & -1.1 \\
\hline 186299 & LHCA5 & "light-harvesting protein of photosystem I"... & -3.5 & -1.4 \\
\hline \multirow[t]{2}{*}{184471} & LHCA1 & "light-harvesting protein of photosystem I"... & -3.1 & -1.3 \\
\hline & \multicolumn{4}{|l|}{ other genes } \\
\hline 189430 & CCP1 & "low-CO2-inducible chloroplast envelope protein"... & -2.5 & -0.7 \\
\hline 194325 & LCl33 & "low-CO2-inducible protein"... & -2.3 & 1.7 \\
\hline 55019 & LCl1 & "low-CO2-inducible membrane protein"... & -2.1 & -2.9 \\
\hline 97127 & EFG1 & "chloroplast elongation factor G"... & -2.9 & -0.6 \\
\hline 135322 & CSP41b & "chloroplast stem-loop-binding protein"... & -2.5 & 0.4 \\
\hline 205573 & CSP41a & "chloroplast stem-loop-binding protein"... & -2.2 & 0.0 \\
\hline 141844 & CDJ4 & "chloroplast DnaJ-like protein"... & 2.6 & 0.8 \\
\hline 134235 & ATPC & "chloroplast ATP synthase gamma chain"... & -2.4 & -0.4 \\
\hline 81427 & $81427 / 1$ & "Chloroplast SRP54 Subunit of Signal Recognition Particle"... & 2.2 & -1.7 \\
\hline 174103 & IPY3 & "soluble inorganic pyrophosphatase"... & -2.3 & -2.8 \\
\hline 148916 & ELI3 & "Early light-inducible protein"... & 2.0 & 2.9 \\
\hline
\end{tabular}

In functional tests of the microarrays we were able to show, besides proof of functionality for the analysis of differential gene regulation under nutrient stress conditions, the deletion of the LHCSR3.1 and LHCSR3.2 in the knockout strain $n p q 4$ [38]. This result let us conclude that the platform is sensitive to investigate knock out or knock down strains. The successful confirmation of the lack of the corresponding transcript and of induction of expression under sulfur stress for LHCSR3.1/ LHCSR3.2 clearly proved the suitability of the array for the analysis of gene deletions. Furthermore, as the probes did not show any cross hybridization or increased unspecific binding, the specificity of the designed probes has clearly been demonstrated in this 


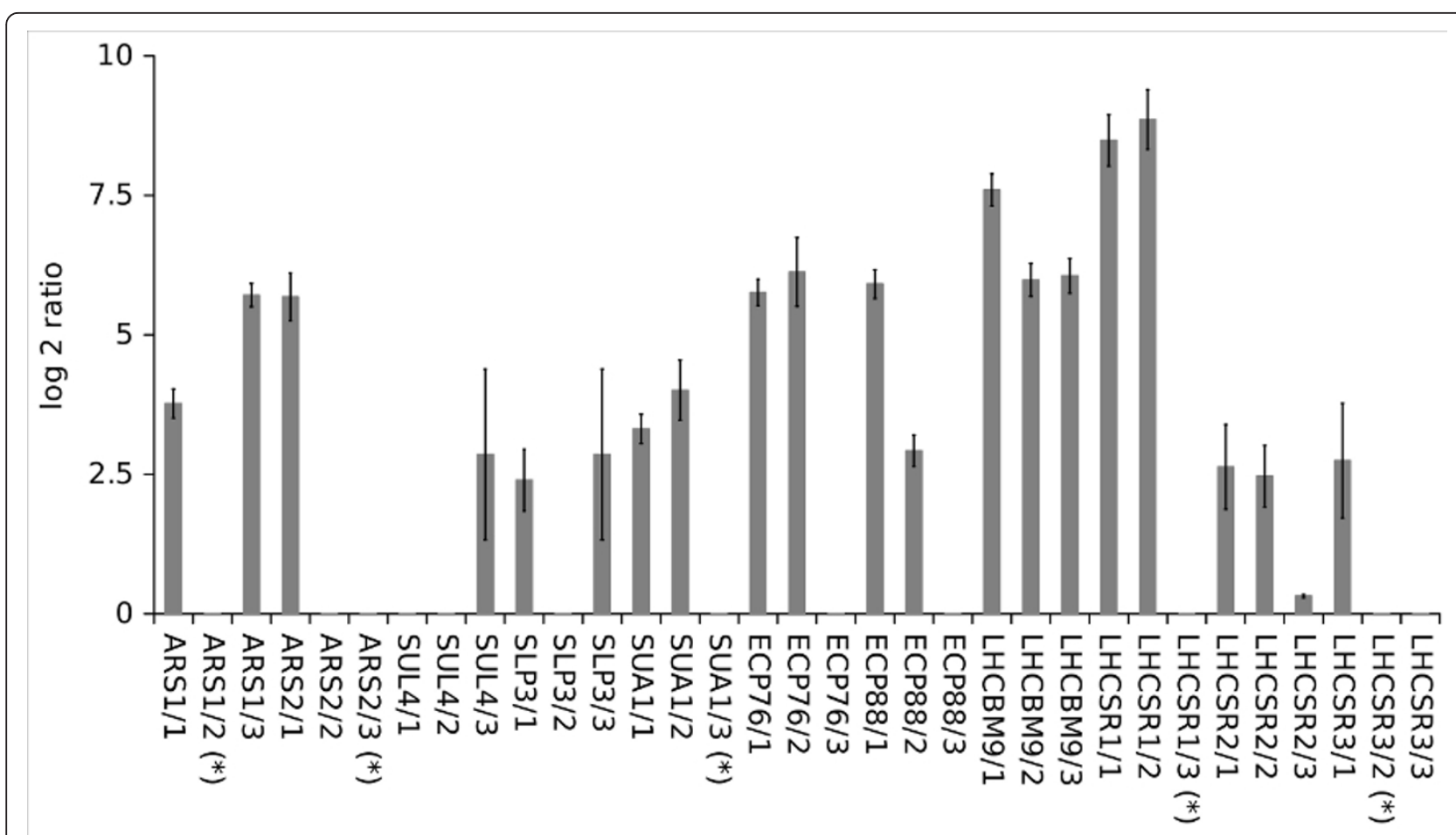

Figure 4 Expression level for known sulfur induced genes in Chlamydomonas reinhardtii, displayed are the average values for 4 time points ( $24 \mathrm{~h}, 48 \mathrm{~h}, 72 \mathrm{~h}, \mathbf{9 6} \mathrm{h}$ ) for the independent probes which showed a significant increased Log 2 ratio for all time points. Probes marked with a star are not specific for the transcript model.

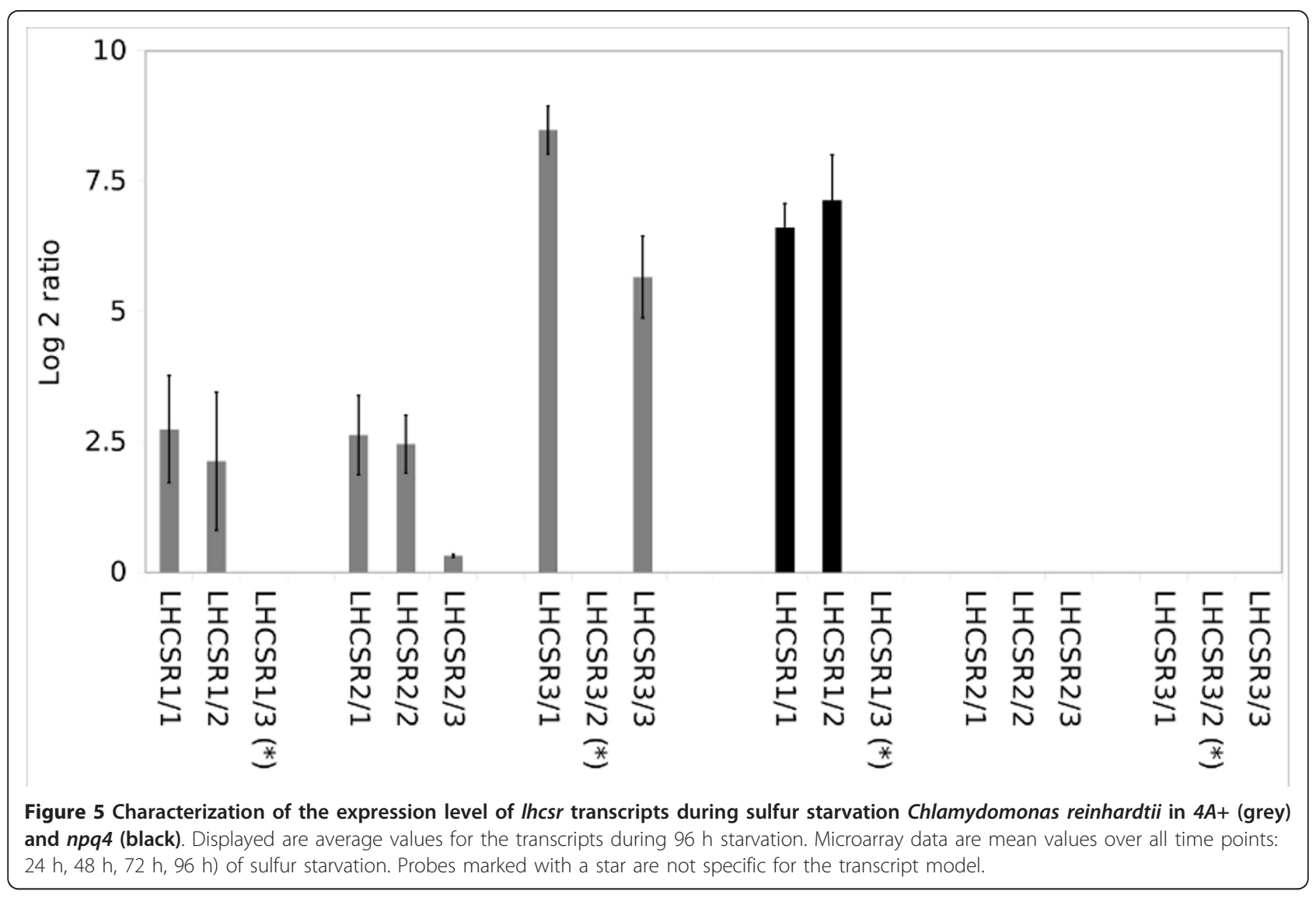


work. Intriguingly, the LHCSR1 genes did not show an up-regulation during nitrogen starvation in our work, thus confirming previous experiments [17].

Under nutrient starvation the first response of the cells is an up-regulation of transport systems required for the specific nutrient. We were able to prove gene expression of such transporters for both, nitrogen and sulfate starvation as well as the increased expression for other nitrogen and sulfate specific genes. Both starvation conditions resulted in an increase of transcript levels already after 12 hours of nutrient depletion. These higher levels of transcription rates were consistent throughout the whole time of the experiment. Differences between our and previous studies [16] could be a result of the longer starvation period and use of different Chlamydomonas strains cc125 and cc3491 instead of D66, ars11, 21gr. Additionally we included into our analysis just those genes with an up-regulation monitored within all time points. Therefore, differences in gene expression level and the number of genes vary.

\section{Conclusion}

In summary, our data proved a high sensitivity of the array as a precondition for further detailed and advanced transcript analyses of mutant $v s$ wt strains in C. reinhardtii. With probes for 14557 transcript models (11000 in AUGUSTUS 10.2) our new array offers a very good coverage of the Chlamydomonas genome. It is noteworthy that current genome annotation predictions estimate around 12000-17000 gene models, so most likely some transcript models are still missing on the platform, however an updated version of the array can easily be established in the future.

\section{Methods}

\section{Genome annotation and Probe design}

DNA sequences for around 15000 predicted transcript models were obtained from the Joint Genome Institute (JGI 3.1 and AUGUSTUS 5.0 http://genome.jgi-psf.org/ chlamy/chlamy.home.html) and several new proteome findings were included. Oligonucleotides that represent the transcriptome of $C$. reinhardtii were designed using Agilent array probe design software (ARRAY EXPRESS ${ }^{\odot}$, see https://earray.chem.agilent.com/earray/) and an open source program (PROMIDE ${ }^{\odot}, \mathrm{CeBiTec}$, Bielefeld). A third probe per transcript was added, which was designed based on the first microarray platform [2]. In summary we used three independent probes per transcript. The expected melting temperature and the $\mathrm{G}+\mathrm{C}$ content for all probes were determined as described in [39]. The microarrays were produced by Agilent $t^{\odot}$ and are available under the array number 024664 . The determined probe sequences were mapped to the newer transcript models using BLAST alignments [40]. A probe with a sequence aligning perfectly to only one transcript sequence, not aligning to any other transcript sequence while allowing for up to 3 mismatches, was considered specific to that transcript model.

The Chlamydomonas full genome microarray (Agilent ${ }^{\odot}$ no.: 024664) matrix, sequences, row data and normalized data are deposited in the GEO database http:// www.ncbi.nlm.nih.gov/projects/geo/ with the accession number (GSE33042).

\section{RNA preparation}

Samples taken from bioreactors $(300 \mathrm{~mL})$ were immediately centrifuged $83000 \mathrm{~g}, 2$ minutes at room temperature). Fresh cell pellets were lyzed immediately with RNA Lysis Buffer and RNA was isolated as previously described [20].

\section{Microarray preparation and data acquisition}

C. reinhardtii microarray slides (Agilent ${ }^{\odot} 4 \times 44 \mathrm{k}$, no: 024664) were used for the transcript analysis. RNA labeling (Quick RNA amplification and labeling kit; Agilent) and microarray hybridization $\left(16 \mathrm{~h}\right.$ at $\left.60^{\circ} \mathrm{C}\right)$ were carried out according to the supplied manual.

\section{Microarray scanning and data analysis}

The microarrays were washed after hybridization according to the Agilent ${ }^{\odot}$ manual, dried in a centrifuge and scanned with a $5 \mu \mathrm{m}$ resolution in a high resolution Agilent ${ }^{\odot}$ DNA microarray scanner. Data extraction was achieved using the feature extraction software (10.7.3.1; Agilent ${ }^{\odot}$ ) and data were normalized and analyzed using EMMA2, an open source software application for microarray data analysis [32,41]. We used a robust normalization method (lowess) and we performed significance tests within all experiments and considered only those probes showing a significant change in their expression (p-values smaller than 0.05). To account for the multiple testing situations, all computed $\mathrm{p}$-values were corrected using the method of Holm-Bonferroni [32,41]. To further limit our result set, we included in our analysis only those genes that showed at least a two-fold up- or down-regulation.

\section{Strains}

The following C. reinhardtii strains were used: wild type cc125 and cc3491. The non-photochemical quenching mutant npq4 was generated by insertional mutagenesis [38], resulting in a knockout of the LHCSR3.1 and LHCSR3.2 genes [33]. The $4 A+$ wild-type strain [42] was used as the control in experiments involving npq4.

Cultures were grown in normal TAP media till early stationary phase and re-suspended after washing (3 times) in TAP minus $\mathrm{S}$ or $\mathrm{N}$ media and cultivated for $96 \mathrm{~h}$ under constant light $\left(200 \mu \mathrm{E} \mathrm{m}^{-2} \mathrm{~s}^{-1}\right)$. 


\section{Samples collections}

Samples from C. reinhardtii cc3491 from five time points were collected at $0 \mathrm{~h}, 24 \mathrm{~h}, 48 \mathrm{~h}, 72 \mathrm{~h}$ and $96 \mathrm{~h}$ after sulfur/nitrogen starvation (T0, T1, T2, T3 and T4 respectively). Reference samples (T0) were harvested from early stationary phase cultures of the corresponding strain before starvation. $n p q 4$ and $4 A+$ were cultivated under sulfate starvation conditions and samples were taken every $24 \mathrm{~h}$ for $96 \mathrm{~h}$.

\section{Additional material}

Additional file 1: Table S1:Transcript specific sequences for the new Chlamydomonas reinhardtii full genome microarray. Probe identifier, probes sequence and origin for the Chlamydomonas reinhardttii $4^{*} 44 \mathrm{~K}$ Agilent microarray.

Additional file 2: Table S2. Confirmation of the Sequence specificity compared to the newest annotation (Augustus 10.2). Specificity for 60 mer oligonucleotides designed for the Chlamydomonas reinhardttii $4 * 44 \mathrm{~K}$ Agilent microarray with three replicates per transcript model in comparison to AUGUSTUS 10.2 annotation (Description: single: Probe is specific for one specific transcript in the used annotation version, multiple: Probe is not specific to one transcript, but to multiple transcripts in the used annotation version. None: probe is not specific to any transcripts in the used transcriptome version.). A summary for all transcript models with the corresponding specific probes (up to three independent probes per transcript) is given in a second Table.

Additional file 3: Table S3. Gene expression of Chlamydomonas reinhardtii under nitrogen starvation. Comparison between RNA-seq [17] (48 h) and microarray data for Chlamydomonas reinhardtii cultures incubated under nitrogen starvation conditions ( 24 h, 48 h, 72 h, 96 h)

Additional file 4: Table S4. Gene expression of Chlamydomonas reinhardtii under sulfur starvation. Comparison between RNA-seq [16] $(48 \mathrm{~h})$ and microarray data for Chlamydomonas reinhardtii cultures incubated under sulfur starvation conditions ( 24 h, 48 h, 72 h, 96 h).

Additional file 5: Table S5. Gene expression of Chlamydomonas reinhardtii under nutrient starvation. Transcript levels for genes with differential expression in Chlamydomonas reinhardtii cultures incubated under sulfur and nitrogen starvation conditions ( 24 h, 48 h, 72 h, 96 h).

\section{Acknowledgements}

The authors would like to thank the German Federal Ministry of Science (BMBF ForSys Partner grant contract 0315265A) for financial support and Prof. Krishna Niyogi from the University of California for providing the npq4 and $4 A+$ mutants. We acknowledge support of the publication fee by Deutsche Forschungsgemeinschaft and the Open Access Publication Funds of Bielefeld University.

\section{Author details}

${ }^{1}$ Algae Biotechnology \& Bioenergy, Dept. of Biology, Center for Biotechnology (CeBiTec), Bielefeld University, 33615 Bielefeld, Germany. ${ }^{2}$ Computational Genomics, Center for Biotechnology (CeBiTec), Bielefeld University, 33615 Bielefeld, Germany. ${ }^{3}$ AG Bioinformatics, GoFORSYS, Max Planck Institute of Molecular Plant Physiology, 14476 Potsdam-Golm, Germany.

\section{Authors' contributions}

JT carried out the microarray design, the sulfur stress experiments and drafted the manuscript. SPA and AG performed the statistical analysis, SA carried out the sequence alignment. MIR and KR participated in the sulfur and nitrogen stress experiments. OK conceived of the study, and participated in its design and coordination and helped to draft the manuscript. All authors read and approved the final manuscript."
Received: 30 June 2011 Accepted: 25 November 2011

Published: 25 November 2011

\section{References}

1. Eberhard S, Jain M, Im CS, Pollock S, Shrager J, Lin Y, Peek AS, Grossman AR: Generation of an oligonucleotide array for analysis of gene expression in Chlamydomonas reinhardtii. Curr Genet 2006, 49(2):106-124.

2. Voss B, Meinecke L, Kurz T, Al-Babili S, Beck CF, Hess WR: Hemin and magnesium-protoporphyrin IX induce global changes in gene expression in Chlamydomonas reinhardtii. Plant Physiol 2011, 155(2):892-905.

3. Merchant SS, Prochnik SE, Vallon O, Harris EH, Karpowicz SJ, Witman GB, Terry A, Salamov A, Fritz-Laylin LK, Maréchal-Drouard L, et al: The Chlamydomonas genome reveals the evolution of key animal and plant functions. Science 2007, 318(5848):245-250.

4. Jain M, Shrager J, Harris EH, Halbrook R, Grossman AR, Hauser C, Vallon O: EST assembly supported by a draft genome sequence: an analysis of the Chlamydomonas reinhardtii transcriptome. Nucleic Acids Res 2007, 35(6):2074-2083

5. Feng L, Liu H, Liu Y, Lu Z, Guo G, Guo S, Zheng H, Gao Y, Cheng S, Wang J, et al: Power of deep sequencing and agilent microarray for gene expression profiling study. Mol Biotechnol 2010, 45(2):101-110.

6. Fu X, Fu N, Guo S, Yan Z, Xu Y, Hu H, Menzel C, Chen W, Li Y, Zeng R, et al: Estimating accuracy of RNA-Seq and microarrays with proteomics. BMC Genomics 2009, 10:161.

7. Nagalakshmi U, Waern K, Snyder M: RNA-Seq: a method for comprehensive transcriptome analysis. Curr Protoc Mol Biol 2010, Chapter 4, Unit 4.11.11-13.

8. Tang F, Barbacioru C, Nordman E, Li B, Xu N, Bashkirov VI, Lao K, Surani MA: RNA-Seq analysis to capture the transcriptome landscape of a single cell. Nat Protoc 2010, 5(3):516-535.

9. van Vliet $\mathrm{AH}$ : Next generation sequencing of microbial transcriptomes: challenges and opportunities. FEMS Microbiol Lett 2010, 302(1):1-7.

10. Wilhelm BT, Landry JR: RNA-Seq-quantitative measurement of expression through massively parallel RNA-sequencing. Methods 2009, 48(3):249-257.

11. Smith DR, Quinlan AR, Peckham HE, Makowsky K, Tao W, Woolf B, Shen L, Donahue WF, Tusneem N, Stromberg MP, et al: Rapid whole-genome mutational profiling using next-generation sequencing technologies. Genome Res 2008, 18(10):1638-1642.

12. Marioni JC, Mason CE, Mane SM, Stephens M, Gilad Y: RNA-seq: an assessment of technical reproducibility and comparison with gene expression arrays. Genome Res 2008, 18(9):1509-1517.

13. Bradford JR, Hey Y, Yates T, Li Y, Pepper SD, Miller CJ: A comparison of massively parallel nucleotide sequencing with oligonucleotide microarrays for global transcription profiling. BMC Genomics 2010, 11:282

14. Liu S, Lin L, Jiang P, Wang D, Xing Y: A comparison of RNA-Seq and highdensity exon array for detecting differential gene expression between closely related species. Nucleic Acids Res 2011, 39(2):578-588.

15. Prochnik SE, Umen J, Nedelcu AM, Hallmann A, Miller SM, Nishii I, Ferris $P$, Kuo A, Mitros T, Fritz-Laylin LK, et al: Genomic analysis of organismal complexity in the multicellular green alga Volvox carteri. Science 2010, 329(5988):223-226

16. González-Ballester D, Casero D, Cokus S, Pellegrini M, Merchant SS, Grossman AR: RNA-seq analysis of sulfur-deprived Chlamydomonas cells reveals aspects of acclimation critical for cell survival. Plant Cell 2010, 22(6):2058-2084.

17. Miller R, Wu G, Deshpande RR, Vieler A, Gärtner K, Li X, Moellering ER, Zäuner $S$, Cornish AJ, Liu B, et al: Changes in transcript abundance in Chlamydomonas reinhardtii following nitrogen deprivation predict diversion of metabolism. Plant Physiol 2010, 154(4):1737-1752.

18. Moseley JL, Chang CW, Grossman AR: Genome-based approaches to understanding phosphorus deprivation responses and PSR1 control in Chlamydomonas reinhardtii. Eukaryot Cell 2006, 5(1):26-44.

19. Mus F, Dubini A, Seibert M, Posewitz MC, Grossman AR: Anaerobic acclimation in Chlamydomonas reinhardtii: anoxic gene expression, hydrogenase induction, and metabolic pathways. J Biol Chem 2007, 282(35):25475-25486.

20. Nguyen AV, Thomas-Hall SR, Malnoë A, Timmins M, Mussgnug JH, Rupprecht J, Kruse O, Hankamer B, Schenk PM: Transcriptome for photobiological hydrogen production induced by sulfur deprivation in 
the green alga Chlamydomonas reinhardtii. Eukaryot Cell 2008, 7(11):1965-1979

21. Ledford HK, Chin BL, Niyogi KK: Acclimation to singlet oxygen stress in Chlamydomonas reinhardtii. Eukaryot Cell 2007, 6(6):919-930.

22. Im CS, Zhang Z, Shrager J, Chang CW, Grossman AR: Analysis of light and $\mathrm{CO}(2)$ regulation in Chlamydomonas reinhardtii using genome-wide approaches. Photosynth Res 2003, 75(2):111-125.

23. Doebbe A, Keck M, La Russa M, Mussgnug JH, Hankamer B, Tekçe E, Niehaus K, Kruse O: The interplay of proton, electron, and metabolite supply for photosynthetic $\mathrm{H}_{2}$ production in Chlamydomonas reinhardtii. J Biol Chem 2010, 285(39):30247-30260.

24. Timmins M, Thomas-Hall SR, Darling A, Zhang E, Hankamer B, Marx UC, Schenk PM: Phylogenetic and molecular analysis of hydrogen-producing green algae. J Exp Bot 2009, 60(6):1691-1702.

25. Melis A, Zhang L, Forestier M, Ghirardi ML, Seibert M: Sustained photobiological hydrogen gas production upon reversible inactivation of oxygen evolution in the green alga Chlamydomonas reinhardtii. Plant Physiol 2000, 122(1):127-136.

26. Melis A, Happe T: Hydrogen production. Green algae as a source of energy. Plant Physiol 2001, 127(3):740-748.

27. Chen M, Zhao L, Sun YL, Cui SX, Zhang LF, Yang B, Wang J, Kuang TY, Huang F: Proteomic analysis of hydrogen photoproduction in sulfurdeprived Chlamydomonas cells. J Proteome Res 2010, 9(8):3854-3866.

28. Saito T, Matsuda Y: Isolation and characterization of Chlamydomonas temperature-sensitive mutants affecting gametic differentiation under nitrogen-starved conditions. Curr Genet 1991, 19(2):65-71.

29. James GO, Hocart $\mathrm{CH}$, Hillier W, Chen $\mathrm{H}$, Kordbacheh F, Price GD, Djordjevic MA: Fatty acid profiling of Chlamydomonas reinhardtii under nitrogen deprivation. Bioresour Technol 2011, 102(3):3343-3351.

30. Bölling C, Fiehn O: Metabolite profiling of Chlamydomonas reinhardtii under nutrient deprivation. Plant Physiol 2005, 139(4):1995-2005.

31. Yang YH, Dudoit S, Luu P, Lin DM, Peng V, Ngai J, Speed TP: Normalization for CDNA microarray data: a robust composite method addressing single and multiple slide systematic variation. Nucleic Acids Res 2002, 30(4):e15.

32. Dondrup M, Albaum SP, Griebel T, Henckel K, Jünemann S, Kahlke T, Kleindt CK, Küster H, Linke B, Mertens D, et al: EMMA 2-a MAGE-compliant system for the collaborative analysis and integration of microarray data. BMC Bioinformatics 2009, 10:50

33. Peers G, Truong TB, Ostendorf E, Busch A, Elrad D, Grossman AR, Hippler M, Niyogi KK: An ancient light-harvesting protein is critical for the regulation of algal photosynthesis. Nature 2009, 462(7272):518-521.

34. Bonente G, Ballottari M, Truong TB, Morosinotto T, Ahn TK, Fleming GR, Niyogi KK, Bassi R: Analysis of LhcSR3, a protein essential for feedback de-excitation in the green alga Chlamydomonas reinhardtii. PLOS Biol 2011, 9(1):e1000577.

35. Wobbe L, Blifernez O, Schwarz C, Mussgnug JH, Nickelsen J, Kruse O: Cysteine modification of a specific repressor protein controls the translational status of nucleus-encoded LHCII mRNAs in Chlamydomonas. Proc Natl Acad Sci USA 2009, 106(32):13290-13295.

36. Beckmann J, Lehr F, Finazzi G, Hankamer B, Posten C, Wobbe L, Kruse O: Improvement of light to biomass conversion by de-regulation of lightharvesting protein translation in Chlamydomonas reinhardtii. J Biotechnol 2009, 142(1):70-77.

37. Blifernez O, Wobbe L, Niehaus K, Kruse O: Protein arginine methylation modulates light-harvesting antenna translation in Chlamydomonas reinhardtii. Plant J 2011, 65(1):119-130.

38. Niyogi KK, Bjorkman O, Grossman AR: Chlamydomonas Xanthophyll Cycle Mutants Identified by Video Imaging of Chlorophyll Fluorescence Quenching. Plant Cell 1997, 9(8):1369-1380.

39. Gordon PM, Sensen CW: Osprey: a comprehensive tool employing novel methods for the design of oligonucleotides for DNA sequencing and microarrays. Nucleic Acids Res 2004, 32(17):e133.

40. Altschul SF, Madden TL, Schäffer AA, Zhang J, Zhang Z, Miller W, Lipman DJ: Gapped BLAST and PSI-BLAST: a new generation of protein database search programs. Nucleic Acids Res 1997, 25(17):3389-3402.

41. Dondrup M, Hüser AT, Mertens D, Goesmann A: An evaluation framework for statistical tests on microarray data. J Biotechnol 2009, 140(1-2):18-26.

42. Dent RM, Haglund CM, Chin BL, Kobayashi MC, Niyogi KK: Functional genomics of eukaryotic photosynthesis using insertional mutagenesis of Chlamydomonas reinhardtii. Plant Physiol 2005, 137(2):545-556. doi:10.1186/1471-2164-12-579

Cite this article as: Toepel et al:: Construction and evaluation of a whole genome microarray of Chlamydomonas reinhardtii. BMC Genomics 2011 12:579.

\section{Submit your next manuscript to BioMed Central and take full advantage of:}

- Convenient online submission

- Thorough peer review

- No space constraints or color figure charges

- Immediate publication on acceptance

- Inclusion in PubMed, CAS, Scopus and Google Scholar

- Research which is freely available for redistribution 